\title{
The Continuing Evolution of Women's Health
}

When the Women's Health Initiative (WHI) and its related studies were designed, it was hoped that hormone therapy (HT) could accomplish multiple goals for women: symptom relief at the menopause, primary prevention of cardiovascular disease and osteoporosis, and even secondary prevention once women had developed disease. The recently published estrogen-only arm of the $\mathrm{WHI}^{1}$ has reported again that global assessments of benefits and risks do not support the use of HT for reaching these manifold goals. The general internist seeking to promote as long, healthy, independent, and meaningful a life as possible must again consider how to address these aims separately.

In this issue of Journal of General Internal Medicine, several articles highlight the importance of alternative strategies to long-term health among women. The exploration of behavioral factors associated with menopause in the paper by Riley et al. is timely. ${ }^{2}$ In a well-conducted survey of a community-based population, they report that lower body mass index (BMI) and moderate alcohol use are associated with lower occurrence of bothersome hot flashes among perimenopausal women, and high dietary fat with lower occurrence in postmenopausal women. The separate examination of women according to their stage of menopause is a strength of this study and may ultimately have important treatment implications. Also notable is the low rate of bothersome hot flashes (34\%), with most described as mild.

Certainly, some of the most difficult results of the WHI studies to interpret and apply in clinical practice are the cardiac outcomes from long-term HT. Studies have been reported with two levels of statistical significance with resulting ambiguity in the interpretation of results. ${ }^{1,3}$ Domains of uncertainty persist, specifically with regard to the effect of age on cardiac outcomes. In a well-conducted meta-analysis of randomized trials, Salpeter et al. explore the possibility of a mortality benefit among younger women using HT. ${ }^{4}$ Although a mortality benefit is reported, no benefit in cardiac outcomes is seen. Thus, little support is found for the proposed biologic mechanisms-through cardioprotective effects of HT before early vascular damage ensues. Nor is there strong support for this hypothesis in the estrogen/progestin arm of the WHI, although there may be a nonstatistically significant trend in the age subgroup analyses reported thus far from the estrogen-only arm. ${ }^{1}$ Nonetheless, because of the high mean age in the WHI (63 years), the door for further exploration of this hypothesis remains ajar.

For women who go on to develop osteoporosis or cardiac disease, effective secondary prevention strategies exist but are underused. In an exploration of guidelines and adoption of bone mineral prevention testing, Morris et al. highlight the low use of bone mineral density (BMD) testing, an important step in the prevention of postmenopausal osteoporotic fractures. ${ }^{5}$ In this study, only $8 \%$ of women 810 with a previous fracture underwent BMD testing. Although the lack of consensus among osteoporosis guidelines is raised as a possible explanation, the National Osteoporosis Foundation, ${ }^{6}$ the American Association of Clinical Endocrinologists, ${ }^{7}$ and the U.S. Preventive Services Task Force ${ }^{8}$ agree that women 65 and older should be screened. Also noted by Morris et al. is the effect of BMD report content and format on physician's interpretation and subsequent interventions. $^{9}$

Cardiac rehabilitation programs foster comprehensive risk factor modification to aid secondary prevention, an intervention philosophically in keeping with generalist practice. However, in a study of use and racial disparities in the use of cardiac rehabilitation programs, Allen et al. report that less than $20 \%$ of women who had a recent myocardial infarction were referred for cardiac rehabilitation. ${ }^{10}$ Reasons for low adoption of accepted strategies by physicians have been recently explored in the Journal and may include knowledge, attitudes, incentives, and systems issues. ${ }^{11}$

To the extent that knowledge and attitudes are barriers to physician adoption of primary and secondary prevention strategies, education is part of the solution. Wayne and DaRosa report practicing clinicians' evaluations of both the relevance and adequacy of instruction in women's health that they received during residency, using as a framework a cognitive and procedural curriculum published by the Federated Council for Internal Medicine. ${ }^{12}$ Whereas basic gynecology (Pap smears, vaginitis) scored well, cognitive areas including osteoporosis and menopausal symptoms were among those with the largest discrepancies between relevance (high) and adequacy of instruction (low). Such studies can help guide the design of women's health curriculum in the coming years.

The articles in this issue of JGIM challenge us to continue examining evidence in the field of women's health and to apply clinical strategies proven to enhance quality of life over a woman's lifetime. The lifespan of women in the United States offers many such opportunities; a 50-yearold woman has a life expectancy of 32 years, a 65-year-old woman of 19 years, and an 80-year-old woman of 9 years. ${ }^{13}$ Responding to this challenge will require further work. Preventive care lends itself well to such technological innovations as reminders ${ }^{14}$ and risk prediction tools. ${ }^{15}$ However, improving care for older women, whose conditions may be complex ${ }^{16}$ and chronic, requires an understanding of patients' and our own sometimes conflicting attitudes toward aging, disease prevention, and risk. ${ }^{17-19}$ There are multiple and repeated points on the continuum of women's lives where preventive interventions are beneficial, but we have much to learn about how best to incorporate these approaches into our clinical practice.-MARILYN M. SCHAPIRA, MD, MPH and JOAN NEUNER, MD, MPH, Division of General Internal Medicine, Medical College of Wisconsin, Milwaukee, Wis. 


\section{REFERENCES}

1. Anderson GL, Limacher M, Assaf AR, et al. Effects of conjugated equine estrogen in postmenopausal women with hysterectomy: the Women's Health Initiative randomized controlled trial. JAMA. 2004;291:1701-12.

2. Riley EH, Inui TS, Kleinman K, Connelly MT. Differential association of modifiable health behaviors with hot flashes in perimenopausal and postmenopausal women. J Gen Intern Med. 2004;19:740-6.

3. Manson JE, Hsia J, Johnson KC, et al. Estrogen plus progestin and the risk of coronary heart disease. $N$ Engl $J$ Med. 2003;349:523-34.

4. Salpeter SR, Walsh JME, Greyber E, Ormiston TM, Salpeter EE. Mortality associated with hormone replacement therapy in younger and older women: a meta-analysis. J Gen Intern Med. 2004; 19:791-804.

5. Morris CA, Cabral D, Cheng H, et al. Patterns of bone mineral density testing: current guidelines, testing rates, and interventions. J Gen Intern Med. 2004;19:783-90.

6. National Osteoporosis Foundation. Physicians' Guide to Prevention and Treatment of Osteoporosis. Washington, DC: National Osteoporosis Foundation; 1999.

7. Hodgson SF, Watts NB, Bilezikian JP, et al. American Association of Clinical Endocrinologists 2001 medical guidelines for clinical practice for the prevention and management of postmenopausal osteoporosis. Endocr Pract. 2001;7:293-312.

8. U.S. Preventive Services Task Force. Screening for osteoporosis in postmenopausal women: recommendations and rationale. Ann Intern Med. 2002;137:526-8.

9. Stock JL, Waud CE, Coderre JA, et al. Clinical reporting to primary care physicians leads to increased use and understanding of bone densitometry and affects the management of osteoporosis. A randomized trial. Ann Intern Med. 1998;128(12 pt 1):996-9.

10. Allen JK, Scott LB, Stewart KJ, Young DR. Disparities in women's referral to and enrollment in outpatient cardiac rehabilitation. J Gen Intern Med. 2004;19:747-53.

11. Heidenreich PA. Understanding and modifying physician behavior for prevention and management of cardiovascular disease. J Gen Intern Med. 2003;18:1060-1.

12. Wayne DB, DaRosa DA. Evaluating and enhancing a women's health curriculum in an internal medicine residency program. J Gen Intern Med. 2004;19:754-9.

13. Arias E, Smith BL. Deaths: preliminary data for 2001. Natl Vital Stat Rep. 2003;51:1-44.

14. Litzelman DK, Dittus RS, Miller ME, Tierney WM. Requiring physicians to respond to computerized reminders improves their compliance with preventive care protocols. J Gen Intern Med. 1993;8:311-7.

15. Sheridan S, Pignone M, Mulrow C. Framingham-based tools to calculate the global risk of coronary heart disease: a systematic review of tools for clinicians. J Gen Intern Med. 2003;18:1039-52.

16. Tierney WM, Overhage JM, Murray MD, et al. Effects of computerized guidelines for managing heart disease in primary care. J Gen Intern Med. 2003;18:967-76.

17. Sarkisian CA, Lee-Henderson MH, Mangione CM. Do depressed older adults who attribute depression to "old age" believe it is important to seek care? J Gen Intern Med. 2003;18:1001-5.

18. Ubel PA, Jepson C, Asch DA. Misperceptions about beta-blockers and diuretics: a national survey of primary care physicians. J Gen Intern Med. 2003;18:977-83.

19. Salkeld G, Cameron ID, Cumming RG, et al. Quality of life related to fear of falling and hip fracture in older women: a time trade off study. BMJ. 2000;320:341-6. 